\title{
La Adquisición del Proceso Lector en Lengua Extranjera
}

\author{
Acquiring Reading Skills in a Foreign Language
}

Inma Piquer *

Universitat de Barcelona

\begin{abstract}
La lectura en lengua extranjera supone un reto y una necesidad para los ciudadanos de una sociedad plurilingüe. Para que este proceso pueda realizarse con éxito, el profesorado debe facilitar a sus estudiantes contextos e instrumentos que permitan la práctica de lecturas colectivas dialogadas, debates, tertulias literarias, trabajo cooperativo y que les acompañe a lo largo de todo el desarrollo de la competencia lectora.
\end{abstract}

Descriptores: Lectura colectiva, Diálogo, Acompañamiento, Trabajo cooperativo.

Reading in a foreign language means both, a challenge and a need to citizens from a plurilingual society. In Catalonia, plurilingualism has been an educational priority and a social aim for several decades. To guarantee the success of this procedure, teachers have the responsibility to provide their students with contexts and resources like dialogic reading, debates, literary gatherings, cooperative work and other collaborative activities for guiding students and help them to achieve reading literacy.

Keywords: Collective reading, Dialogue, Guidance, Cooperative work.

\section{Introducción}

La competencia comunicativa, vista desde una perspectiva global, incluye procesos orales y dialógicos, estrategias de lecto-escritura y desarrollo de dominios metalingüísticos. En este amplio espectro de habilidades, la competencia lectora se destaca como uno de los ámbitos más significativos con las que cuenta el aprendiz en el proceso de adquisición de lenguas extranjeras.

En Cataluña, donde conviven dos lenguas oficiales de forma institucional en el mundo educativo, se enseñan también una o más lenguas extranjeras en el currículum de la enseñanza obligatoria y se planifican actuaciones para que parte del alumnado recién llegado conserve también su lengua de origen. En este contexto, podemos decir que el plurilingüismo de los estudiantes es una generalización y aprender a leer en diferentes lenguas es una necesidad. Desafortunadamente, en las aulas, parte del profesorado de lengua extranjera tiende a limitar los materiales, la oferta de textos que ofrece a sus estudiantes y la propuesta de actividades de lectura, debido a la falsa creencia que los

*Contacto: mpiquer3@gmail.com

ISSN: $1696-4713$

rinace.net/reice/

revistas.uam.es/reice
Recibido: $\quad 14$ de septiembre 2014

$1^{\text {a }}$ Evaluación: 13 de diciembre de 2014

$2^{\text {a }}$ Evaluación: 18 de marzo 2015

Aceptado: $\quad 24$ de abril 2015 
textos reales son demasiado complejos para el aprendizaje. Este hecho condiciona notablemente la predisposición de los estudiantes a aprender a leer en diferentes lenguas, con lo cual, difícilmente llegan a hacerlo por placer, en especial porque los textos "preparados" o "simplificados" a menudo presentan escasos retos cognitivos y se alejan de la espontaneidad de los textos auténticos.

Las tecnologías digitales pueden paliar esta dificultad dado que favorecen el acceso a múltiples documentos, en formatos diversos, con hipervínculos de ampliación y búsquedas explicativas. Las situaciones de aprendizaje escolar con soporte digital estimulan la autonomía del alumnado cuando éste puede tomar decisiones sobre itinerarios de exploración lectora, y puede gestionar la inmediatez de la consulta en tesauros y diccionarios en línea y el acceso a traductores plurilingües. Las propuestas sistemáticas de un plan lector en el centro educativo deben incluir una oferta rica y variada de textos en diferentes formatos, la realización de actividades de lectura cooperativa e individual dentro y fuera de las aulas y la explotación de todos los recursos digitales educativos al alcance del centro escolar, con el objetivo de generar escenarios de aprendizaje que permitan a los estudiantes adquirir el hábito lector en diferentes lenguas.

La lectura autónoma y la comprensión profunda representa todo un reto que precisa que el profesorado investigue, descubra y presente a los estudiantes nuevos formatos de lectura motivadores, actividades comunicativas, debates literarios, y otras iniciativas creativas para estimular la lectura (Grabe y Stoller, 2011). El objetico es presentar diversas metodologías utilizadas en la enseñanza de la lectura en lengua extranjera, que permitirán al profesorado guiar los pasos de sus estudiantes desde que "comienzan a leer", hasta que llegan a "disfrutar leyendo" en una lengua extranjera.

\section{Aprendizaje de la Lectura}

Para Siegel (1992), las lenguas varían entre sí dependiendo de las reglas de correspondencia grafema-fonema (CGF). Según estas reglas las lenguas pueden ser de ortografía transparente, cuando a cada grafema le corresponde siempre el mismo sonido, o bien de ortografía opaca cuando esta correspondencia no siempre se da y existe una falta de regularidad. Partiendo de este hecho, el lector puede aprender a leer según las directrices que marca el método alfabético o bien siguiendo las que marca el método global:

- Método alfabético: También denominado Bottom-up método de aprendizaje de la lectura. Según este método, el lector lee partiendo de la letra, la palabra y la frase en orden progresivo (Gough, 1972).

- Método global: También denominado Top-down método de aprendizaje de la lectura. Parte de la presunción de que el lector interroga el texto a partir de sus conocimientos previos con el fin de procesarlo y comprenderlo. La creación de expectativas y la respuesta a las cuestiones que se va planteando le permiten deducir el contenido.

El método global gozó de gran popularidad a partir de los años 70 (Goodman, 1967). Según el autor, el lector cuenta con tres fuentes de recogida de información: el conocimiento semántico (conocimiento del mundo), el sintáctico (conocimiento de la 
estructura del lenguaje) y el grafo-fonético (conocimiento de la interrelación sonidografema).

\subsection{El aprendizaje de la lectura en lengua extranjera}

La hipótesis tradicional de interdependencia lingüística (Cummins, 1979) predecía que los niños que presentan problemas en el aprendizaje de la L1, los presentarán también en el de la L2 y el de la LE. Esta propuesta se ha visto contestada por algunas experiencias de lectura en estudiantes que aprendían lenguas con alfabetos muy diferenciados, como el chino y el francés o el inglés (Moore, 2010). Por otro lado, los estudios parecen confirmar que la transferencia de los logros se da con mayor profusión que la transferencia de errores, de modo que todas aquellas habilidades académicas logradas con éxito en L1 se transferirán con mayor facilidad a la L2, L3 y cualquier otra lengua que aprendan los estudiantes.

Cuando se habla de lectura en lengua extranjera (L2, L3 y Ln), Goodman (1967) destacaba el hecho de que el grado de fluidez en lengua extranjera del lector condiciona su velocidad lectora en dicha lengua. Se constata que a más conocimiento de la lengua extranjera, más fluidez lectora y menos dependencia de los aspectos grafo-fonéticos de la lengua.

Para Gibbons (2002) el lector competente en lengua extranjera debe ser capaz de:

- Conocer el código en el que lee (Code breaker).

- Participar del texto a partir de sus conocimientos culturales previos (Texto Participante). El vínculo entre lengua y cultura es inseparable. El conocimiento de una lengua va directamente asociado a la cultura de la que forma parte. Así pues, no podemos deducir que el lector competente en L1 lo será también en lengua extranjera si no hay algún contacto explícito y suficiente con la cultura de la lengua en cuestión.

- Ser usuario del texto (Text user). En función de las expectativas puestas en el texto, el lector sabrá seleccionar el método de lectura más adecuado. Ejemplos de métodos de lectura rápida o extensiva son el Skimming y el Scanning. El primero se realiza con la finalidad de decidir el interés que puede tener una lectura más intensiva, el segundo permite localizar una información concreta en un texto.

- Analizar el texto (Text Analyst). La lectura en lengua extranjera precisa mucho más que de traducciones literales, precisa de razonamientos mentales complejos, y de una actitud crítica y analítica ante el texto que le permita hacer deducciones y leer entre líneas.

Ejemplos de actividades que permiten al niño convertirse en un lector analítico en lengua extranjera podrían ser: la selección de lecturas placenteras en diferentes formatos (cómics, revistas, storybird, y más), la lectura en voz alta en pareja y en grupos, el conocimiento y la alternancia de los métodos de lectura alfabético y global en el proceso lector, el conocimiento de las 2.000 palabras básicas que constituyen el $80 \%$ de los textos en lengua extranjera, etcétera (Mikulecky, 2008).

La habilidad lectora integrada en el desarrollo comunicativo del estudiante escolarizado tiene un sentido instruccional básico (se aprende a leer y se aprende a aprender), y constituye un proceso orgánico de enriquecimiento competencial. Sin embargo, la 
realización de actividades de forma aislada y no progresiva ni pautada dificultará el hecho de que el proceso de lectura en lengua extranjera por parte del aprendiz se realice con éxito. El conocimiento del profesorado de lenguas extranjeras de métodos y estrategias dirigidas al impulso de la habilidad lectora permite que dicho proceso se realice con éxito en el aula para que pueda tener después continuidad fuera de ella.

Leer fuera de los entornos escolares puede ser especialmente enriquecedor en aspectos vivenciales, ya sea en situaciones de aprendizaje informal (exploración de carteles de metro o autobús, o lectura esporádica de folletos publicitarios o de instrucciones mecánicas, por poner algún ejemplo) o no formal (estudios para sacarse el permiso de conducción, cursillos de verano, o clases de mejora deportiva). Las actividades escolares que permiten establecer continuidad y referencias cruzadas entre estos entornos, pueden llegar a ser en ocasiones altamente motivadoras para el aprendizaje lector en situaciones reales (como por ejemplo las lecturas para la preparación de actividades deportivas y extraescolares; acciones de voluntariado; prácticas pre-profesionales; aprendizaje en servicio y servicio comunitario).

\subsection{De la lectura en lengua extranjera colectiva a la individual}

Cualquier método de impulso a la lectura en lengua extranjera, para que tenga éxito, debe ir del plano interpersonal al intrapersonal favoreciendo la prolepsis del lector (Rogoff y Gardner, 1984). La prolepsis es el proceso que permite al aprendiz pasar de depender de un mediador experto para poder realizar una actividad, a realizarse de forma individual y autónoma.

El paso del plano interpersonal al intrapersonal es fundamental en cualquier proceso lector. Dixon y Krauss (1996) lo ejemplificaban a partir de la figura del niño que aprende a leer en su L1 conjuntamente con el adulto que lee las páginas del libro mientras su rol se limita a escuchar, seguir con el dedo y repetir algunas palabras. Poco a poco, el niño empieza a leer en voz alta primero y de forma silenciosa después, convirtiéndose con el tiempo en un lector adulto competente. Un lector para quien el lenguaje impreso se acaba convirtiendo en una herramienta que le ayuda a estructurar el pensamiento. A lo largo de todo este proceso de aprendizaje las actividades orales que permiten la comunicación entre los interlocutores en torno a la lectura tendrán una doble función, la de aprender a leer y también la de mejorar la producción oral (Geva, 2006).

Así pues, se puede afirmar que pese a la calidad del documento objeto de lectura en L2 seleccionado, las actividades previas y posteriores, el conocimiento de la L2 por parte del lector, el entorno creado, etcétera, no se tendrá la garantía de éxito en la comprensión profunda del documento si no se conoce y se sigue todos los pasos inherentes a la lectura en lengua extranjera. Ésta requiere procesos asociativos propios de la transferencia intercultural, sin los cuales leer en una segunda o tercera lengua no llega a los niveles de profundidad que requiere la comprensión metalingüística. Los estudios sobre lectura cooperativa realizados por Anne Marie Palincsar y Ann Brown en los años ochenta en Estados Unidos inspiraron múltiples estudios en este campo. Entre ellos destacan todos aquellos que encontraron en las posibilidades digitales una fuente inagotable de inspiración y búsqueda (Vaughn, Kligner y Briant, 2001).

Volviendo al centro de interés, la lectura en lengua extranjera, cabe citar los estudios realizados por Michael Cole en el campo de la lectura cooperativa en lengua extranjera y analizar detenidamente su Método QAR (Question, Asking, Reading). Este método de 
lectura cooperativa en lengua extranjera permite a estudiantes con dificultades en la lectura, incluso en su L1, leer de forma eficaz en lengua extranjera. Se trata de un método de lectura en grupo que parte de: la selección de textos interesantes y claramente estructurados, la distribución de roles específicos entre los diferentes miembros del grupo, el establecimiento de un tema de debate y apoyo, y el guiado de una persona experta (Cole, 2003).

El método QAR se basa en la creación de grupos de lectura, partiendo de la ideología de pasar de la lectura interpersonal a la intrapersonal siguiendo una serie de pasos concretos: (1) lectura en voz alta alternando lectores a criterio del experto, (2) asignación de roles por parte del experto y lectura individual silenciosa teniendo en cuenta el rol asignado, (3) conversación en grupo sobre la lectura a partir de los roles asignados, y (4) cambio de roles y repetición de la actividad (1). La implementación de este método de lectura en lengua extranjera precisa de la utilización de instrumentos para que pueda ser eficaz, sobre todo con los aprendices más jóvenes y noveles. Además del texto, se recomiendo también utilizar tarjetas con los roles, guión de la sesión, hojas de papel y lápices para tomar notas dependiendo del rol asignado, etcétera. Los métodos de lectura colectiva en lengua extranjera la presentan como una actividad colaborativa, dramatizada y perfectamente pautada que despierta en los lectores actitudes positivas (Buescher, 2009).

\section{Métodos de Lectura Colectiva}

En Cataluña se han desarrollado y se desarrollan diferentes métodos de lectura colectiva. Los grupos interactivos, las tertulias literarias dialógicas, la lectura entre iguales o los padrinos de lectura son ejemplos destacados. Los métodos de lectura colectiva se basan en el principio del aprendizaje dialógico. Las escuelas, las comunidades de aprendizaje, las bibliotecas, entre otras, son el contexto idóneo para esta práctica y el voluntariado, las familias, los expertos y los iguales la fuente de recursos humanos imprescindible. A continuación se habla de todos estos aspectos con detalle.

\subsection{El aprendizaje dialógico y los grupos interactivos}

La construcción de significados y la adquisición de conocimiento no pueden realizarse de forma individual, se debe hacer de forma colectiva y dialógica. Es imprescindible que la comunicación forme parte de las primeras etapas del proceso lector en lengua extranjera. Las habilidades comunicativas son las que permiten a los interlocutores resolver muchos problemas y situaciones que no podrían resolverse de forma individual. Partiendo de esta situación comunicativa, que se desarrolla a su vez dentro de un contexto sociocultural determinado, se produce un tipo de aprendizaje llamado aprendizaje dialógico. Es imprescindible que este aprendizaje respete en términos de igualdad a todos los miembros del grupo.

Así pues, en una situación de lectura colectiva dialógica en lengua extranjera, a pesar de que el rol de liderazgo recaiga en el profesor de lengua extranjera o persona más experta del grupo, los miembros integrantes de éste se sienten en igualdad de condiciones respecto a sus compañeros. El bagaje cultural que aportan a la lectura dialógica los diferentes miembros del grupo, sus intereses y motivaciones personales, así como la creación de un clima de lectura distendido y solidario, permitirán que cualquier actividad 
de lectura colectiva en lengua extranjera se convierta en un acto constructivo y motivador para todos sus miembros.

En el contexto educativo de aula, cuesta imaginar la manera de llevar a cabo actividades de lectura colectiva basadas en el aprendizaje dialógico. Los profesionales de la enseñanza de todo el mundo han visto en la creación de grupos interactivos (GI) y bancos de voluntarios la respuesta a muchas de sus necesidades, ya que favorecen las interacciones para que los estudiantes alcancen los aprendizajes propuestos. Para conseguirlo, el profesorado crea grupos heterogéneos, dependiendo de la edad, la complejidad de la tarea asignada, las características del grupo o la disponibilidad de recursos y, con el apoyo de voluntarios (en la mayor parte de los casos), se gestiona el trabajo asignado a cada grupo, de tal forma que cada uno de ellos queda al mando de uno de los grupos.

Para cada grupo, el profesor planifica y presenta una actividad diferente que deben realizar todos los miembros del grupo durante un tiempo determinado bajo la supervisión de la persona voluntaria. Una vez transcurrido el tiempo acordado, los grupos cambian de actividad hasta haberlas realizado todas. Al finalizar la sesión, todos los estudiantes habrán realizado todas las tareas propuestas (Píriz, 2011). La planificación de tareas diseñadas para el trabajo en grupos interactivos debe ser cuidadosa. Trabajar en GI no consiste en agrupar estudiantes heterogéneamente para que trabajen de forma más controlada, se trata de favorecer la interacción entre los miembros del grupo para que la realización de la tarea sea producto de la comunicación y de los acuerdos tomados. En todo este proceso, la figura del voluntario juega un papel muy importante.

\subsection{El voluntariado en centros}

El voluntario, además de coordinarse con el profesor, antes y después de las sesiones para conocer las actividades a realizar y proponer opciones de mejora, también promueve la interacción entre los diferentes miembros del grupo y el resto de grupos de la clase. Ejemplos de perfiles de voluntariado idóneo son: estudiantes de prácticas, lectores estudiantes de ciclos formativos, bachillerato y universitario de turno de tarde, familiares de estudiantes, ex -estudiantes, profesorado emérito, etcétera. En Cataluña existe un banco de voluntarios especializado en apoyar la lectura en los centros educativos. El nombre de esta organización es LECXIT y tiene por objetivo incrementar el éxito educativo de los niños a través del trabajo para la mejora de su comprensión lectora.

En cuanto a mejorar el rendimiento de los estudiantes en lengua inglesa en los centros, destaca el proyecto que se desarrolla en la isla de Menorca. Elena Serra, Asesora de Lenguas Extranjeras del Centro de Profesorado de Menorca, ha creado y coordina un banco de voluntarios integrado por personas jubiladas de habla inglesa que han fijado su residencia en Menorca y que colaboran con el profesorado de inglés de la isla de forma comprometida y periódica. Las diferentes asociaciones británicas (clubes) y los centros piloto se han organizado para participar en esta iniciativa, quienes se han hecho eco en las publicaciones locales de la isla y se destaca por su implicación activa la llamada Menorca Britannia.

Además del voluntariado, la investigación universitaria es otro ejemplo de acciones de impulso a la lectura. Asociaciones como GRAI (Grupo de Investigación sobre 
Aprendizaje entre Iguales) de la Universidad Autónoma de Barcelona se dedican a estudiar nuevas fórmulas de mejora de la competencia y comprensión lectora de los estudiantes. Muchos de los centros que trabajan con grupos interactivos y que cuentan con bolsas de voluntarios son las denominadas Comunidades de Aprendizaje. Las comunidades de aprendizaje, a pesar de encontrarse dentro del marco educativo legal, responden a una ideología y organización interna muy concretas donde la participación de toda la comunidad a través del voluntariado es el eje vertebrador. Es en las comunidades de aprendizaje donde se recupera la filosofía de las Tertulias Literarias Dialógicas realizadas con adultos en los años 80 con la intención de impulsar la lectura en las aulas de educación infantil, primaria y secundaria.

\subsection{Las tertulias literarias dialógicas}

Las Tertulias Literarias Dialógicas, inspiradas en las lecturas sociales de los Ateneos Libertarios de principios del siglo XX, se consolidaron como herramienta educativa institucional en 1980, en la escuela de adultos de "La Verneda - Sant Martí" de Barcelona, desde donde se popularizaron gracias a CONFAPEA (Confederación de Federaciones y Asociaciones de Personas Adultas en Educación y Cultura Democrática de Personas Adultas) y su posteriormente a portal digital (CONFAPEA, 2010). Los primeros precedentes de estas tertulias se encuentran en el nacimiento de las "Sociedades Económicas de Amigos del País" en el siglo XVIII. Estas sociedades fueron creadas con la finalidad de ofrecer cursos de alfabetización y conversación en horario nocturno para los trabajadores y gente sin recursos (Pallares, 2004).

En la actualidad, son muchos los profesionales de la enseñanza que han adoptado este modelo de lectura colectiva adecuándolo a su realidad de aula, porque aporta espacios de relación social, facilita la expresión oral, mejora la interacción personal y desarrolla la escucha activa, a partir de la lectura profunda y reflexiva de documentos de actualidad y obras de la herencia literaria universal. Así pues, se encuentran múltiples ejemplos de tertulias literarias dialógicas desarrolladas en centros de educación primaria y secundaria donde los estudiantes formulan hipótesis y hacen interpretaciones sobre el texto leído colectivamente, sin ceñirse necesariamente a las intenciones del autor, sino partiendo de las apreciaciones o valoraciones personales y la asociación de ideas.

Las tertulias literarias dialógicas fomentan el diálogo textual en grupo, el análisis crítico, la comunicación argumentativa y la reflexión, todas ellas habilidades lectoras complejas, difícilmente alcanzables sin un trabajo de acompañamiento lector previo. Este trabajo de guía, seguimiento y refuerzo es imprescindible que parta de la acción tutorial y de la atención individualizada, permitiendo a los estudiantes adquirir las capacidades básicas de velocidad y comprensión lectora. Las tertulias literarias dialógicas fomentan el diálogo textual en grupo, el análisis crítico y la reflexión, todas ellas habilidades lectoras complejas difícilmente alcanzables sin un trabajo de acompañamiento lector previo. Este trabajo de guía, seguimiento y refuerzo es imprescindible que parta de la acción tutorial y de la atención individualizada, permitiendo a los estudiantes adquirir las capacidades básicas de velocidad y comprensión lectora.

A continuación se presentarán dos ejemplos de lectura cooperativa por parejas, la lectura en pareja entre iguales y los padrinos de lectura. 


\subsection{La lectura en pareja}

En España, la lectura en pareja entre iguales en L1 y L2 se ha convertido en una práctica cada vez más habitual en los centros. Sin embargo, en el campo de la lectura en lengua extranjera, a pesar que la investigación así lo diga y la apuesta de la administración exista, hay que esperar antes de obtener resultados de calidad en lectura cooperativa.

Opportunities for co-construction of knowledge through collaborative dialog may Involve both teacher-learner ( TL ) and learner-learner (LL ) interacciones (Philp y Tognini, 2009, p. 47). La lectura en pareja es una práctica fácil de integrar dentro del horario escolar. No implica ninguna medida organizativa especial ya que se realiza en el aula y los miembros participantes son los mismos estudiantes y el profesor (Durán, 2012).

El profesor debe hacer parejas heterogéneas, de tal forma que uno de los dos tenga un grado superior de competencia lectora con respecto a su compañero. De esta forma, uno de ellos podrá tomar el rol de tutor y el otro de tutorado. Las primeras sesiones de lectura en pareja comienzan con una breve explicación del profesor quien presentará las diferentes actividades a realizar durante la sesión. Una vez hechas las explicaciones y aclaradas las dudas será el momento de trabajar en pareja.

En primer lugar, procede que el tutor formule preguntas sobre el contenido del texto a leer. De esta forma se pone en marcha el diálogo y se crean expectativas sobre el texto. Después comienza la lectura en voz alta. Se recomienda que el tutor comience a leer y, unos instantes después, lo hará también el estudiante imitando su modelo de dicción y pronunciación. A continuación, el estudiante volverá a leer el texto en voz alta bajo la supervisión del tutor. Este ofrecerá sus servicios de corrección siempre que sea necesario siguiendo la técnica PPP (pausa, pista y ponderación). Consiste en detener al estudiante cuando se detecte que éste ha cometido un error, darle tiempo para que la subsane, orientarlo con una pista si no se sale y, por último, valorar positivamente su esfuerzo por mejorar.

Una vez finaliza la lectura en voz alta, juntos revisan las preguntas formuladas inicialmente. Por último, el estudiante procede a leer el texto en voz alta una vez más para mostrar que ha hecho suyo el texto. Esta técnica de lectura permite al profesor ofrecer el apoyo y soporte de calidad a sus estudiantes en el proceso lector. Es por ello que se pueden encontrar prácticas de lectura en parejas en primaria y prácticas de lectura en pareja en secundaria.

Su simplicidad y viabilidad la hacen perfectamente extrapolable a la clase de lengua extranjera para potenciar la lectura en L3 y L4, o siguientes, siempre y cuando se tomen las medidas necesarias para que pueda llevarse a cabo con éxito. Entre estas medidas, destaca el hecho de introducir la lectura en lengua extranjera de forma progresiva, siempre dependiendo del grado de competencia alcanzado en la lengua o lenguas propias. Por otra parte, dada la importancia que tienen los primeros estadios de este proceso y la necesidad de que el tutor sea un lector competente en lengua extranjera, la lectura entre iguales se convierte en un método poco fiable cuando hablamos de lectura en L3 y L4. Así pues, al menos durante las primeras etapas de adquisición del proceso lector, hay que pensar en otras técnicas más fiables como las de los padrinos de lectura que se presentan a continuación. 


\subsection{Los padrinos de lectura}

Una variante de la lectura en pareja son los padrinos de lectura. Esta es una práctica que se está llevando a cabo con éxito en muchos centros de educación primaria y secundaria de Cataluña. En la mayor parte de los casos, atendiendo a las necesidades organizativas de los centros, los apadrinamientos se realizan entre estudiantes del mismo centro. Niños y niñas de cursos superiores apadrinan un compañero de cursos iniciales. Sin embargo, algunos centros han visto en esta práctica una oportunidad única para facilitar la buena relación inter-centros primaria-secundaria y la han incorporado a su programa de actividades de traspaso. El procedimiento empleado en esta práctica coincide con el de la lectura en pareja. El "padrino" supervisa el proceso de adquisición lector del "ahijado " siguiendo los pasos descritos en el subapartado anterior. La intimidad y conocimiento mutuo que se crea entre los dos miembros mediante la realización de esta actividad trasciende las aulas y permite crear lazos afectivos sólidos y duraderos.

Los objetivos que se plantea la propuesta lectora de los "padrinos de lectura" se alcanzan de forma prácticamente natural. Además del objetivo de mejorar la competencia lectora del alumnado tutorado, hay que añadir el de ofrecer oportunidades de calidad a los "padrinos" que les permiten aprender enseñando (Garriga y Oller, 2012). Los estudiantes tutorados disfrutan del hecho de disponer de un estudiante mayor que ellos durante todo el proceso. Este hecho les motiva a: esforzarse, respetar al padrino asignado, tratar de complacerle con las mejoras conseguidas y disfrutar de la amistad que trasciende a la simple realización de esta actividad.

Los estudiantes padrinos valoran muy positivamente el hecho de aprender mientras enseñan. El hecho de preparar las lecturas, de tener que explicar, evaluar y corregir obliga a reflexionar, empatizar y adecuar la ayuda a las necesidades mostradas por el estudiante en cada momento. Todo un proceso de aprendizaje y sensibilidad que fomenta el crecimiento intelectual, personal y emocional. En cuanto al planteamiento de propuestas de lectura en lengua extranjera se valora el camino recorrido en lengua propia y se hace una planificación de apoyo que permita su implementación en los centros.

\section{Conclusiones}

Siguiendo las directrices del Marco de Acción para el aprendizaje de lenguas extranjeras, el Departament d'Ensenyament, en Cataluña ha diseñado y desarrollado un plan de impulso a la lectura para mejorar la comprensión lectora del alumnado en catalán, castellano, así como en las demás lenguas del currículum oficial.

Esta acción se hace también extensiva más allá de las asignaturas lingüísticas y se interesan por el trabajo lector a las diferentes áreas curriculares que el alumnado cursa, en cualquiera de las lenguas de instrucción. Metodologías como AICLE (Aprendizaje Integrado de Contenidos y Lengua Extranjera) se enriquecen con la gestión de textos en diferentes soportes y de tipologías muy diversas y permite estimular la oralidad en escenarios de debate, en entornos de interacción social y en procesos de aprendizaje cooperativo y de autogestión del conocimiento.

En conclusión, podríamos decir que las técnicas de lectura colectiva, gestionadas adecuadamente, permiten pasar del plano interpersonal al intrapersonal para que el placer por el diálogo, el debate argumentativo y el intercambio social, llegue a generar el 
gusto por la lectura individual y silenciosa, la reflexión profunda y el respeto por las distintas sensibilidades lectoras. Esta transferencia puede producirse de forma colaborativa en cualquier lengua y cualquier cultura, y permite apreciar la riqueza de la literatura universal.

\section{Referencias}

Buescher, K. (2009). Reading in a second language classroom: a pedagogical report on sociocultural strategies for reading texts in the elementary French classoom. Tesis inédita. University of Massachussets.

Cole, M. (2003). Cultural psychology. A once and future discipline. Cambridge, MA: Harvard University Press

Cummins, J. (1979). Linguistic interdependence and the educational development of bilingual children. Review of Educational Research, 49, 221-251.

Dixon-Krauss, L. (1996). Vygotsky's sociocultural perspective on learning and its application to western literacy instruction. En L. Dixon-Krauss, Vygotsky in the classroom. Mediated literacy instruction and assessment (pp. 7-24). White Plains, NY: Longman Publishers.

Duran, D. (2012). Llegim en parella. Escola Catalana, 475, 22-23.

Garriga, A. y Oller, M. (2012). El voluntariat en un programa de tutoria entre iguals per millorar la lectura. Guix, 383, 37-40.

Geva E. (2006). Learning to read in a second language: research, implications, and recommendations for services. En R.E. Tremblay, R.G. Barr, R.V. Peters (Eds.), Encyclopedia on early childhood development. Montreal: Centre of Excellence for Early Childhood Development.

Gibbons, P. (2002). Reading in a second language. En J. Cummins y P. Gibbon (Eds.), Scaffolding language, scaffolding learning: teaching second language learners in the mainstream classroom (pp. 77-100). Portsmouth: Heinemann.

Goodman, K.S. (1967). Reading: a psycholinguistic guessing game. Journal of the Reading Specialist, 6, 126-135.

Gough, P.B. (1972). One second of reading. En F.J. Kavanagh y G. Mattingly (Eds.), Language by ear and by eye. (pp. 331-358). Cambridge, MA: MIT Press.

Grabe, W. y Stoller, E. (2011). Teaching and research reading. Nueva York: Pearson Education.

Mikulecky, B. (2008). Teaching reading in a second language. Recuperado de http://longmanhomeusa.com/

Moore, D. (2010). Multilingual literacies and third script acquisition: young Chinese children in French immersion in Vancouver, Canada. International Journal of Multilingualism, 7(4), 322-342.

Pallarès, V. (2004). Les tertúlies literàries dialògiques: paraules per a transformar. Castellon: Universitat Jaume I.

Philp, J. and tognini, R (2009). Language acquisition in foreign language contexts and the differential benefits of interaction. International Review of applied Linguistics in Language Teaching, 47, 245-266.

Píriz, RM. (2011). Una experiencia de grupos interactivos en un centro de secundaria. Tendencias Pedagógicas, 17, 51-64. 
Rogoff, B. y gardner, W. (1984). Adult guidance of cognitive development. En B. Rogoff y J. Lave (Eds.), Everyday cognition: its development in social context (pp. 95-116). Cambridge, MA: Harvard University Press.

Siegel, L. (1992). An evaluation of the discrepancy definition of dyslexia. Journal of Learning Disabilities, 25, 618-229.

Vaughn, S., Klingner, J. y Bryant, D. (2001). Collaborative strategic reading. Remedial and Special Education, 22, 66-74. 